\title{
Creating an Illusion of Movement between the Hands Using Mid-Air Touch
}

\author{
Dario Pittera, Damien Ablart, and Marianna Obrist.
}

\begin{abstract}
Apparent tactile motion (ATM) has been shown to occur across many contiguous parts of the body, such as fingers, forearms and the back. More recently, the illusion has also been elicited on non-contiguous part of the body, such as from one hand to the other when interconnected or not interconnected by an object in between the hands. Here we explore the reproducibility of the intermanual tactile illusion of movement between two free hands by employing mid-air tactile stimulation. We investigate the optimal parameters to generate a continuous and smooth motion using two arrays of ultrasound speakers, and two stimulation techniques (i.e. static vs. dynamic focal point). In the first experiment, we investigate the occurrence of the illusion when using a static focal point, and we define a perceptive model. In the second experiment, we examine the illusion using a dynamic focal point, defining a second perceptive model. Finally, we discuss the differences between the two techniques.
\end{abstract}

Index Terms-Mid-air Touch, Tactile Illusions, Touch, Apparent Tactile Motion, Haptics.

\section{INTRODUCTION}

$\mathbf{T}$ ACTILE feedback is being implemented more and more in modern technology. Vibrotactile controllers are well known in the classic game industry (e.g.: for the Sony PlayStation, Microsoft Xbox, Nintendo Wii U, etc.). Recently, VR companies are also trying to implement tactile feedback in their controllers; such as in the Oculus Rift, the HTC Vive or the Sony VR headsets. Those efforts underline the increased demand for using haptic feedback to make the users' experience more immersive. As of today, tactile feedback is mainly provided statically to the hands (i.e. by means of actuators providing a vibration without any motion pattern), hence limiting the potential of experiencing dynamic situations. For instance, we can imagine being a superhero moving an energetic wave from one hand to the other, feel it growing before throwing it towards an enemy, or we could feel a shock wave moving under our feet after an explosion in a game. Dynamic movement is also something we experience in reallife situations: the wind blowing from one side of our hands and body to the other, the waves of the sea moving from one part to another. Hence, to increase the immersivity in a virtual environment (VE), emerging haptic technologies should be able to provide a tactile sensation of movement to render additional and more realistic experiences.

To achieve a sense of motion we can draw upon research in psychology regarding tactile illusions of movement, such as the apparent tactile motion (ATM). The advantage of using a perceptual illusion to elicit the feeling of motion is that we

All the authors are from the School of Engineering and Informatics, University of Sussex, East Sussex, UK (see www.multisensory.info).

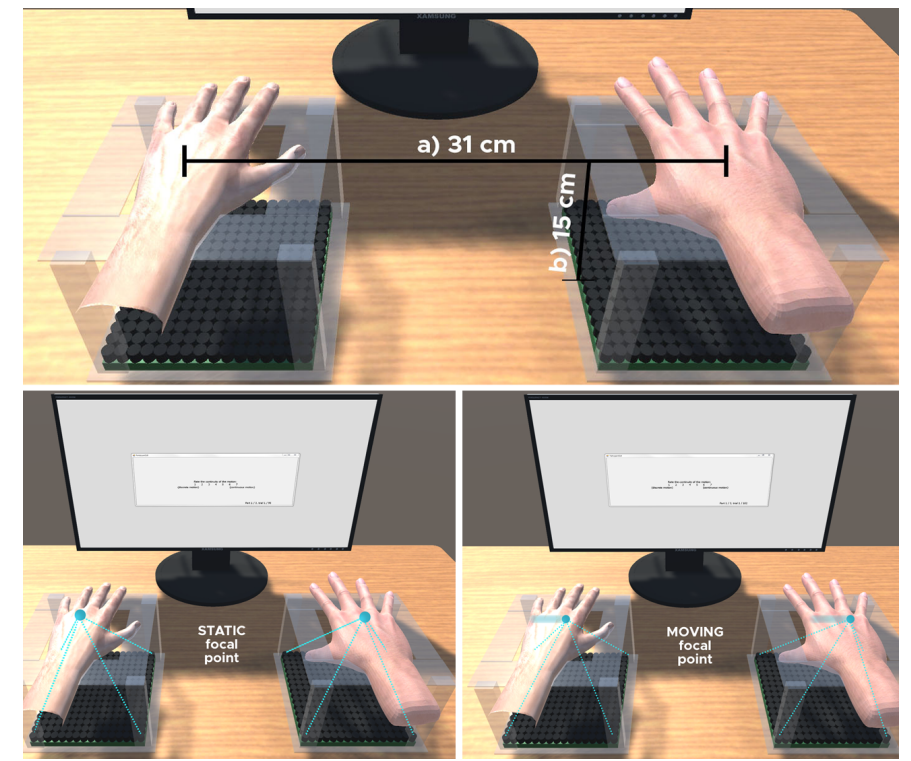

Fig. 1. Experiments setting. TOP: For both experiments, a) the distance between the palm of the two hands was set at $31 \mathrm{~cm}$. b) The hands were laying on the two acrylic boxes at a distance of $15 \mathrm{~cm}$ above the two mid-air haptic devices. LEFT: experiment 1, a static focal point was delivered to the center of the distal part of each palm. RIGHT: experiment 2, a dynamic focal point was delivered on the distal part of the two hands palm.

can achieve the same perceptual sensation using a drastically reduced number of actuators. This will reduce obstruction of the user and the programming complexity. Previous research demonstrated the successful use of the ATM: Israr et al. delivering movement on the back [1], [2], Zhao et al. [3] rendering the movement between two hands interconnected by a tablet, and Pittera et al. [4] investigating the ATM illusion between two non-interconnected hands. All of these studies made use of physical touch.

With the evolution of haptic technology we now have alternative ways to deliver tactile feedback, with the latest innovations enabling its delivery in mid-air. The advantage of using such technology is the possibility of creating and delivering tactile feedback without the need for cumbersome attachments on the user's skin. In this study, we chose to employ a commercially available ultrasound-based device that showed potential for creating a new variety of experiences. This device has been previously used to enhance the user experience of short movies [5] and art exhibitions [6]. Carter et al. [7] employed ultrasound arrays as an input interface, allowing color rendering, pinch-to-zoom interaction, and the possibility of interacting with a web application. Long et al. [8] were able to successfully render volumetric shapes. Obrist 

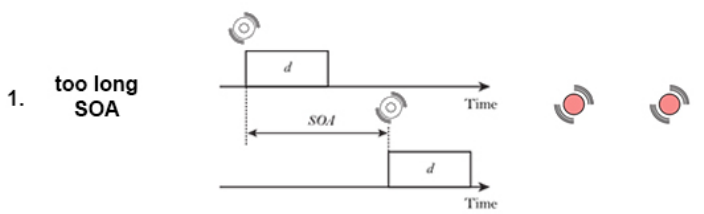

discrete motion

2. right
SOA
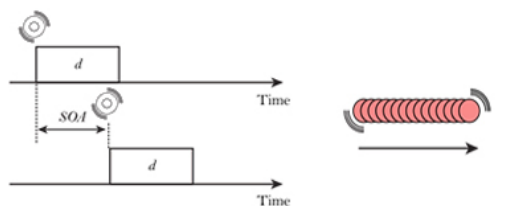

coninuous
motion

3. too short SOA

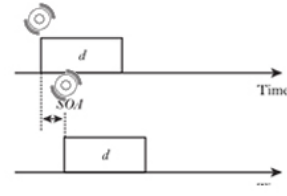

Fig. 2. Representation of the apparent tactile motion, showing the perceptive effect accordingly to different stimulus onset asynchrony (SOA). If the SOA is too long, the perception will result in two discrete vibrations (top). If the SOA is too short, the perception will be merged in a single point (bottom). With an optimal SOA, a motion will be perceived (center).

et al. [9] delivered emotional content on the hand using midair tactile stimulation. Finally, there have also been attempts to replicate the findings of traditional psychological research employing physical touch, using mid-air touch. For instance, Horiuchi et al. [10] replicated the rubber hand illusion using mid-air stimulation and projected images.

With the proliferation of virtual and augmented reality technology, there is an increased interest in creating more immersive and compelling experiences through integrating tactile stimuli. Being scalable, attachment free, and temporally precise, mid-air technology can expand the set of tools designers use to design more immersive and realistic scenarios in traditional console games, VR and AR environments.

We used ultrasound mid-air technology to investigate the ability to recreate the ATM illusion. Having the ability to easily create different tactile patterns, we investigated the optimal values (in terms of frequency, duration, onset time asynchrony (SOA), and direction) needed to render a smooth tactile sensation of movement through 1) a static and 2) a dynamic focal point (see Fig. 11. We provide and compare two perceptive models, one for each technique: static and dynamic point tactile stimulation. Finally, we compare our results with a previous study that used physical tactile stimulation (i.e. vibrotactile actuators) [4]. Although we hypothesize that a dynamic point stimulation will result in a stronger illusion of movement compared to a static point, it is not clear if the results from the dynamic mid-air focal point will differ from those of the physical touch on the hands. Our results suggest that the dynamic mid-air focal point is indeed capable of providing a greater illusion of movement compared to a static point, and it works equally well as physical touch. This work contributes to the basic understanding of midair tactile perception, allowing the representation of more complex scenarios which include tactile movement. With this work, we aim also to provide designers of tactile displays with an understanding of the optimal parameters for the design of a smooth tactile movement.

\section{Previous Research}

The sense of touch is a multifaceted system that pervades the whole body, and comprehends cutaneous inputs from the mechanoreceptors in the skin accounting for pressure, vibration, pain, temperature, and pleasure associated with a certain tactile stimulation. In its broader meaning, haptics, it comprehends all those sensations associated with an active touch (kinesthetic inputs from the muscles, tendons, and joints), providing updated information on the position of the limbs in the space (proprioception), the level of the muscle stretch, but also information on the objects' $3 \mathrm{~d}$ shape and their texture [11]. Hence, rendering the complexity of this system through the modern actuators still represents a challenge. In this paper, we explore the use of tactile illusions as a promising approach to overcome current limitations in creating complex tactile sensations without the need to focus on the precise rendering of its components. In other words, tactile illusions can provide design shortcuts for creating convincing tactile experiences. Here, we are particularly interested in those illusions that can render a tactile sensation of movement.

\section{A. Tactile illusions of movement}

Prior studies of physical touch stimulation identified three main types of illusions of movement using a psychophysical approach [12], [13], [14]: 1) the cutaneous rabbit illusion, 2) the haptic funneling, and 3) the ATM (more recent tactile illusions of movements are described in [15], [16], [17]).

In the cutaneous rabbit illusion, two vibrotactile actuators are modulated in a timely fashion to create a third illusory perceptual sensation like a rabbit hopping in between the two real actuators [18]. In the haptic funneling illusion, two actuators vibrate at different intensity, creating a third perceptual point in-between, whose position will be determined accordingly to the variation of the intensity of the two vibrations [19]. In this study, we focus on the ATM illusion. Here, two actuators are activated while modulating the stimulus-onset asynchrony (SOA) so that the user will perceive a feeling of movement between the two sites of stimulation (see Fig. 2] [20]. There are three possible scenarios: a) if the SOA is too long, then the two vibrations will be perceived as discrete and no illusion of movement will occur (Fig. 2. top). b) If the SOA is too short, the two vibrations will be perceptually merged into a single one and no illusion of movement will occur (Fig. 2, bottom). c) Only when the SOA is optimal will the two vibrations appear as a movement (Fig. 2, center). Many other tactile illusions are being studied, but their description is beyond the scope of this paper (for a more extensive overview on tactile illusions, see [12], [21]).

\section{B. The apparent tactile motion}

The phenomenon of the ATM explained above, has been studied since the early 90s [20], [21], [22], [23], [24]. In 
these pioneering studies, authors investigated the fundamental parameters that allow for a smooth tactile motion. They concluded that there was a significant positive correlation between the duration of the stimulus and the SOA. This same relation was also found in the visual modality version of the apparent tactile illusion (e.g. two static lights turning on and off at a certain frequency providing an effect of motion) [23]. Research on the ATM is ongoing, with different aspects of the phenomenon being studied. Miyazaki et al. explored the illusion on the fingers [25], Lechelt et al. on the forearm [26], and Israr et al. on the back [1], [3]. Zhao et al. investigated the ATM between two hands interconnected by a tablet [3]. Moreover, Pittera et al. [4] recently illustrated how the illusion occurs when the two hands are not interconnected. Finally, Wilson et al. studied the apparent tactile motion occurring on a single hand using mid-air stimulation [27]. These studies have shown the main factors that contribute to a smooth sensation of motion: the stimulus duration and the SOA between the first and the second tactile stimulation.

The apparent tactile illusion can be easily applied to the realm of VR tactile applications. In fact, we could provide the user with a smooth feeling of motion, making the virtual experience even more immersive. Currently, one of the main limitations when providing tactile feedback resides in the attachment of more cumbersome devices, which could break the immersivity in the virtual environment. New emerging technologies such as the mid-air haptic devices could tackle this issue. These devices are capable of delivering tactile stimulation without the need of attachments on the skin (see Section II-C. . Moreover, they allow more control over parameters such as frequency, intensity, direction, ramp up/down of the signal, and signal waveform. However, to date, there is no empirical evidence on their ability to induce the ATM, or if the key variables to obtain a smooth motion are the same as when using physical touch (i.e. duration and SOA). We do not know if the duration and the SOA of the tactile stimuli would still be the main key variables. In this paper, we investigate if mid-air touch can elicit an illusion of motion, and also the parameters we need to control in order to achieve a smooth motion sensation. Since this new technology allows the design of different mid-air tactile patterns, and to increase the chance of eliciting a smoother sensation of movement, we replicate the ATM employing two different tactile patterns: a static focal point and a dynamic focal point.

\section{Mid-air haptic technology}

Current mid-air haptic devices deliver tactile feedback by three main modalities: air, laser and ultrasound. Laser mid-air devices are based on the thermoelastic effect, using indirect laser radiation to convey tactile feedback [28]. One of the drawbacks of this technology is that the user must wear an attachment on the skin to perceive the tactile feedback, and for protection. Airborne technology represents a safe alternative for conveying tactile feedback. Air is pushed through a nozzle and, depending on the specific device employed, can travel for several meters from the source [29]. The main limitation is that air tends to dissipate quickly and even when it is possible to

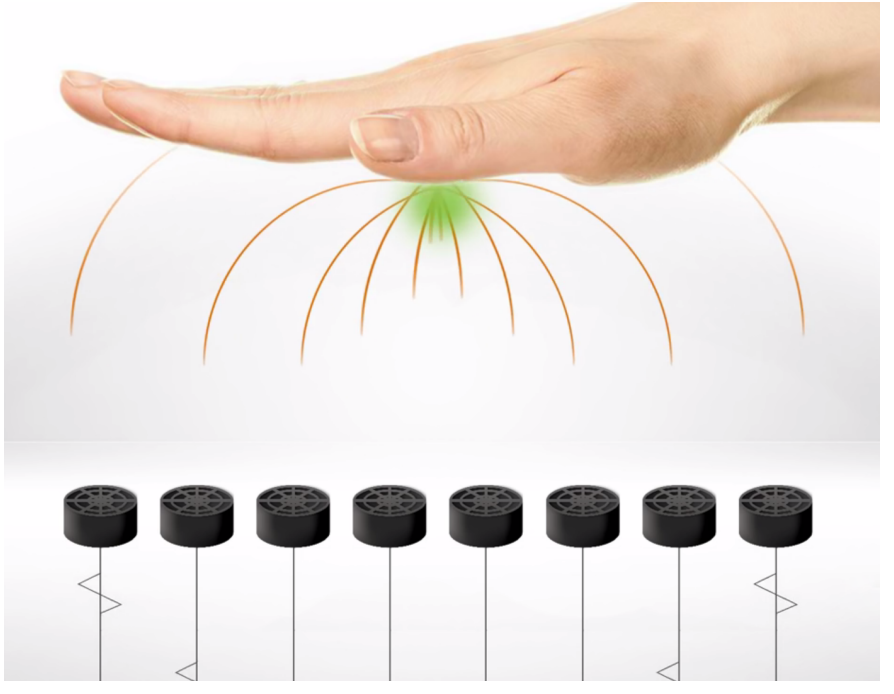

Fig. 3. The ultrasonic technology allows displaying a tactile feedback in midair using a series of ultrasonic transducers emitting sound waves that can be felt when they are spatially and temporally aligned, creating a focal point. (Picture provided by UltraHaptics LtD)

contain the dissipation (i.e. in the case of vortex technology), its spatial resolution is low, making the achievement of a localized sensation impossible [29], [30], [31].

One of the most promising mid-air technologies employs focused ultrasound, exploiting the principle of acoustic radiation force [32], [7]. The ultrasonic technology was originally introduced by Hoshi et al. [32] and it allows the delivery of tactile feedback in mid-air using a series of ultrasonic transducers. These emit sound waves at ultrasonic frequencies that can be felt when they are spatially and temporally aligned, creating a focal point (Fig. 3). Such a setup allows users to experience tactile feedback without any direct contact or attachment to the body. The psychophysical knowledge of this mid-air haptic technology is still in its infancy, but prior work has mapped high-frequency stimulations (i.e. $250 \mathrm{~Hz}$ ) to the Pacinian corpuscles (mechanoreceptors in the glabrous skin, sensitive to high-frequency vibrations from $50 \mathrm{~Hz}$ to 10 $\mathrm{kHz}[33]$ ) and low frequency stimulations (i.e. $16 \mathrm{~Hz}$ ) to the Meissner corpuscles (mechanoreceptors sensitive to low frequency vibrations below $80 \mathrm{~Hz}$ [34]) [35]. The ultrasound midair technology has a greater temporal and spatial resolution (40 kHz, $1 \mathrm{~cm},[8])$ compared to vortex based systems and compressed air systems. It is possible to control the position and the intensity of the focal points at high frequency (16 $\mathrm{kHz}$ ), allowing a multitude of tactile patterns by means of different durations, frequencies, and ramp up/down of the signal. In addition, this technology is safe and scalable.

\section{STUdY SETUP AND APPROACH}

The objective of this study is to investigate the reproducibility of the ATM between two non-interconnected hands using mid-air tactile stimulation. Furthermore, we are interested in knowing whether a static or dynamic focal point will provide the smoothest tactile motion sensation.

In the following two sections we present two studies to explore the optimal parameters needed for creating a smooth 
tactile transition from one hand to the other. Across both experiments, we followed a psychophysical approach to determine the relationship between the mid-air stimuli and the resulting tactile perception (i.e. occurrence of the ATM).

We used two mid-air haptic devices developed by Ultrahaptics Ltd (www.ultrahaptics.com). This device consists of an array of ultrasound speakers $(16 \times 16)$ that allows precise control of the tactile stimuli delivery (e.g., frequency, amplitude, SOA, ramp up/down of the signal, waveform and duration)(see Fig. 1). We programmed a graphical user interface (GUI) in C\# to guide participants through the experiment. The ultrasonic haptic boards were controlled through a program written in $\mathrm{C}++$ and connected to the GUI through the TCP/IP protocol. The boards were synchronized using high precision timers (ms order). The tactile focal points employed in the two experiments were designed using amplitude modulation (i.e. to create a $200 \mathrm{~Hz}$ focal point, the intensity of the point was alternating from $0 \%$ to $100 \%, 200$ times per second). The intensity change followed a sinusoid curve to minimize the noise of the devices. In experiment 1 , we projected a single static focal point onto the distal part of each palm. In experiment 2 , the focal point moved along the distal part of each palm in a straight line, from the right-to-left or left-toright, at different SOAs values. We chose to project the tactile feedback onto the distal part of the palm because, especially for experiment 2, we needed a uniform (flat) area on which to display the focal point. In fact, if the dynamic mid-air point hit the skin at different heights, the perception could be nonuniform and hard to perceive.

Participants were sitting on a chair with their two arms leaning on arm supports and their palms downwards on two boxes (see Fig. 1). The boxes were acrylic structures, each containing a mid-air haptic device, with a rectangular hole of $10 \times 8 \mathrm{~cm}$ in the center, to allow the mid-air stimulation to reach participants' distal part of the palms. The distal part of the palm of each hand was aligned with the center of the boxes' hole, where the mid-air stimulation was provided. The location of the stimulus delivery did not vary with the hand size; the hand was always hit at the center of the distal part of the palm. The boxes were designed to keep the users' hands at a constant distance of $15 \mathrm{~cm}$ above the ultrasound array, which is within the optimal working range of the device. The distance between the palms was kept at $31 \mathrm{~cm}$ as in [4]. Instructions were provided on a screen. Ethics approval for this research was obtained by the University's Science and Technology Ethics committee.

\section{EXPERIMENT 1: TACTILE ILLUSION OF MOVEMENT WITH A SINGLE FIXED FOCAL POINT}

The aim of this experiment was first to investigate whether the ATM between the hands occurs when using a mid-air stimulation. Second, if the illusion does occur, to determine the optimal parameters to elicit a smooth illusion of movement to define a perceptive model. In this first experiment, we investigate the illusion using a single static focal point projected on the distal part of participants' palms.

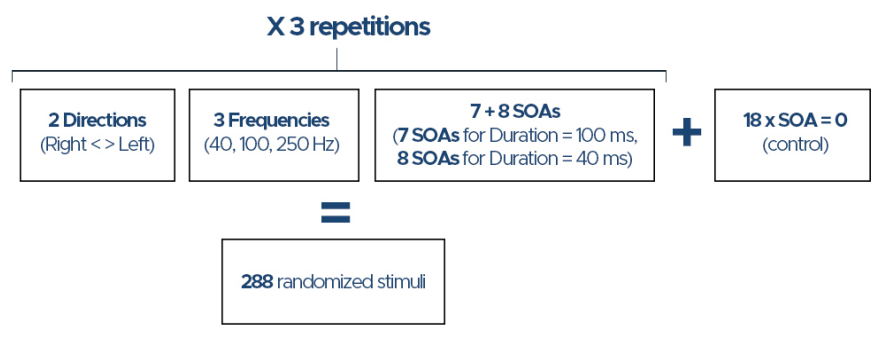

Fig. 4. Experimental design for experiment 1. Every mid-air haptics stimulus was a combination of the four variables (i.e., duration, direction, frequency, SOA), and a control condition with SOA set at 0 , for a total of 288 randomized stimuli. The picture is not representative of the order of presentation of the stimuli.

\section{A. Method}

We first conducted a pilot experiment with 7 participants $(3$ females, age $\mu=27.4, \mathrm{SD} \pm 3.6)$ to determine the frequency and the duration of the mid-air tactile stimulation. Based on previous studies [3], [36], [4], we tested three different frequencies $(70 \mathrm{~Hz}, 100 \mathrm{~Hz}$, and $250 \mathrm{~Hz})$, and two durations (100 ms and $400 \mathrm{~ms}$ ). While testing the smoothness of the motion in our pilot experiment (using the same study set-up as described above, see Fig. 1), the only pair of frequencies that was statistically similar was the $70 \mathrm{~Hz}$ and $100 \mathrm{~Hz}$ (p $>$.5). Hence, for the main experiment we selected only the $100 \mathrm{~Hz}$ and $250 \mathrm{~Hz}$ frequencies. In addition, knowing that the mid-air touch perception is associated not only with the Pacinian corpuscles (receptors for high-frequency vibrations from $50 \mathrm{~Hz}$ to $10 \mathrm{kHz}$ ) but also with the Meissner corpuscles (receptors for low-frequency vibrations $<80 \mathrm{~Hz}$ ) [35], we additionally tested the $40 \mathrm{~Hz}$ frequency, for a total of three frequencies $(40 \mathrm{~Hz}, 100 \mathrm{~Hz}$, and $250 \mathrm{~Hz}$ ).

Based on the pilot study and accounting for the mechanoreceptors relevant for high and low-frequency vibrations, the experimental design for experiment 1 consisted of three blocks of 96 randomized mid-air tactile stimuli, for a total of 288 stimuli.

We chose two stimuli durations (i.e., 100 and $400 \mathrm{~ms}$ ). For each duration, we chose a different set of SOAs, equally divided as in [3], [4]. For the $100 \mathrm{~ms}$ duration the SOAs ranged from $15 \mathrm{~ms}$ to $190 \mathrm{~ms}$ for a total of eight intervals, and for the $400 \mathrm{~ms}$ duration, SOAs ranged from $15 \mathrm{~ms}$ to $350 \mathrm{~ms}$, for a total of seven intervals. These different SOA ranges are required to reach a plausible effect of movement [3], [4]. For each duration, we also added an $\mathrm{SOA}=0$, as a control condition to account for random responses from participants. Every tactile stimulus was set to ramp up and down at a time equal to the $20 \%$ of the stimulus duration as in [3], [4]. Therefore, every stimulus was a combination of duration (100 $\mathrm{ms}$ and $400 \mathrm{~ms}$ ), SOA (the two different sets, plus the control SOA), frequency $(40 \mathrm{~Hz}, 100 \mathrm{~Hz}$, and 250 $\mathrm{Hz}$ ) and direction (from left to right and vice-versa) (see Fig. 4 for an overview).

Before beginning the testing phase, participants had the opportunity to familiarize themselves with the mid-air tactile stimulation. A series of minimum three pairs of stimuli were presented to participants' palm, while the researcher ensured 
that the user understood the experimental procedure. After this training phase, stimuli were presented one at the time, with at least five seconds gap to avoid tactile habituation. After the stimulus occurred, participants were guided by the GUI to report verbally if they felt a sensation of movement between the hands. In the case of a negative answer, the subsequent trial was presented. Instead, if a feeling of motion was reported, the participant was asked to indicate verbally the smoothness of motion on a rating scale visible on the GUI, ranging from 1 (discrete motion) to 7 (continuous motion). Participant's answer were recorded on the computer by the researcher. Additionally, participants could ask to repeat the stimulation. Each block of 96 stimuli was separated by a 2minute break. Participants wore headphones to mask environmental and device noises. Moreover, a beep sound was played through the headphones before the beginning of each trial. Overall, the experiment lasted 45 minutes. All participants were compensated with a $£ 7.5$ voucher for participating in the experiment.

\section{B. Participants}

A total of 20 participants took part in the study ( 9 females, age $\mu=26.8, \mathrm{SD} \pm 7.7)$. They had normal or glasses/lens corrected vision and no history of neurological or psychological disorders. All participants were right-handed. Upon arrival, participants were asked to read the information sheet and sign a consent form, followed by the task explanation.

\section{Results}

To ensure that the rating scale was used appropriately, we checked the ratings for the SOA $=0$ (control trials). The overall ratings were respectively 0.39 and 0.12 for durations of $100 \mathrm{~ms}$ and $400 \mathrm{~ms}$, meaning that participants were not feeling movement when the tactile point was provided at the same time on the two hands. Users' ratings (1, discrete motion to 7 , continuous motion) were averaged for the two durations across participants.
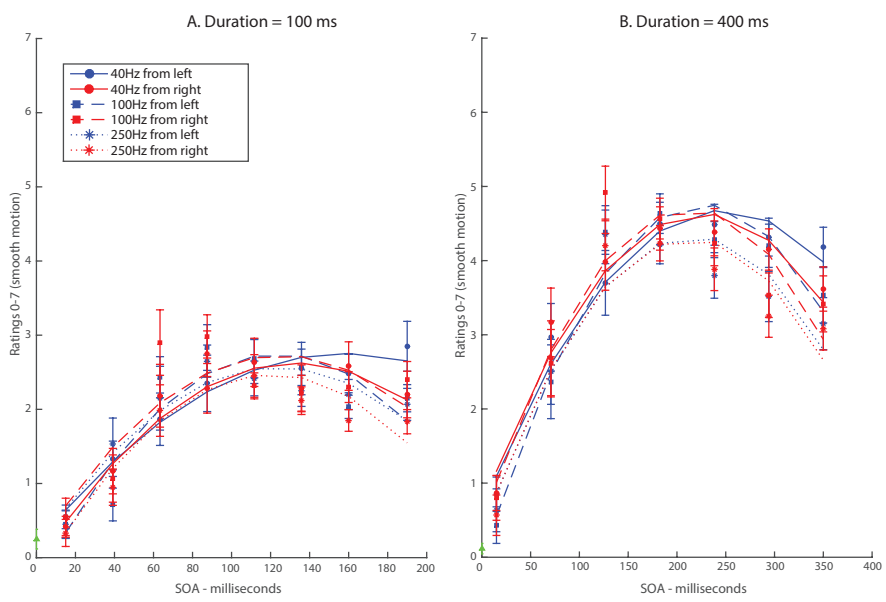

Fig. 5. Plots of the ratings of the illusion of movement (x-axis) per SOAs (y-axis). The left graph shows the plot for $100 \mathrm{~ms}$ duration, and the right graph shows the plot for $400 \mathrm{~ms}$ duration. Dots and lines represent raw data and model fitting, respectively.
TABLE I

OPTIMAL SOA VALUES (IN MS.) AND QUADRATIC FIT $\left(\mathrm{R}^{2}\right)$ FOR THE DIFFERENT COMBINATIONS OF DURATION, FREQUENCY, AND DIRECTION.

\begin{tabular}{lllcc}
\hline Duration & Frequency & Direction & SOA peak $(\mathrm{ms})$. & $\mathrm{R}^{2}$ \\
\hline $100 \mathrm{~ms}$ & $40 \mathrm{~Hz}$ & from left & 158.57 & .95 \\
$100 \mathrm{~ms}$ & $40 \mathrm{~Hz}$ & from right & 123.62 & .83 \\
$100 \mathrm{~ms}$ & $100 \mathrm{~Hz}$ & from left & 123.32 & .89 \\
$100 \mathrm{~ms}$ & $100 \mathrm{~Hz}$ & from right & 133.06 & .94 \\
$100 \mathrm{~ms}$ & $250 \mathrm{~Hz}$ & from left & 125.33 & .66 \\
$100 \mathrm{~ms}$ & $250 \mathrm{~Hz}$ & from right & 120.51 & .86 \\
$400 \mathrm{~ms}$ & $40 \mathrm{~Hz}$ & from left & 247.45 & .96 \\
$400 \mathrm{~ms}$ & $40 \mathrm{~Hz}$ & from right & 226.12 & .95 \\
$400 \mathrm{~ms}$ & $100 \mathrm{~Hz}$ & from left & 216.27 & .89 \\
$400 \mathrm{~ms}$ & $100 \mathrm{~Hz}$ & from right & 226.04 & .96 \\
$400 \mathrm{~ms}$ & $250 \mathrm{~Hz}$ & from left & 213.09 & .86 \\
$400 \mathrm{~ms}$ & $250 \mathrm{~Hz}$ & from right & 213.05 & .90 \\
\hline
\end{tabular}

Fig. 5 illustrates the average ratings as a function of SOA for the two durations, the two directions, and the three frequencies along with best-fit quadratic trends. The two lowest parts of the curves correspond to low SOAs (left part of the curves = merged tactile perception) and to high SOAs (right part of the curves $=$ discrete tactile perception). The peaks of the curves are reported in Table I and they correspond to the optimal values of the SOAs needed to achieve a smooth sense of motion. On average the optimal SOA value was found to be $177.21 \mathrm{~ms}$.

Fig 5 suggests non-linear trends of the rating scores. Moreover, comparing our results with previous research ([3], [32]), we can hypothesize that with very small and very large values of SOA, participants' ratings of the smoothness of motion will decrease. Therefore, a quadratic model seems more appropriate in describing our dataset. Using $\mathrm{R}$ software (v. 3.5.1) with the nlme package, we fit our data to a quadratic model accounting for individual differences between the subjects. Subjects represented our random variable (model 1). Our model, had a $\mathrm{R}^{2}=.52, \mathrm{AIC}^{1}=6541.12$.

By inspecting Fig 5 there seems to be an interaction between the duration and SOA. Hence, we accounted for this interaction in our model. After fitting our dataset into a quadratic function, $\mathrm{y}=$ duration $+\mathrm{SOA}+\mathrm{SOA}^{2}+$ duration:SOA (model 2), the AIC decreased to $6386.93\left(\mathrm{R}^{2}=.56\right)$. A likelihood ratio test between the two models suggested model 2 as more accurate in predicting our data, $\mathrm{p}<.0001$. Therefore our final model is:

$$
\begin{aligned}
& \text { 1) } y=0.47-5 * 10^{-4} * d u r+2 * 10^{-2} * S O A-9 * 10^{-5} * S O A^{2} \\
& +4 * 10^{-5} * \text { dur }: S O A
\end{aligned}
$$

where the colon (:) represents an interaction. In this experiment we investigated the optimal parameters to achieve a

\footnotetext{
${ }^{1}$ The AIC (Akaike information criterion) is a parameter used to compare different models, the smaller the value between two models, the better the model fits the data (F. Korner-Nievergelt, T. Roth, S. von Felten, J. Guelat, B. Almasi, and P. Korner-Nievergelt (2015). Chapter 11 - Model Selection and Multimodel Inference, in Bayesian Data Analysis in Ecology Using Linear Models with R, BUGS, and STAN, pp. 175-196.).
} 


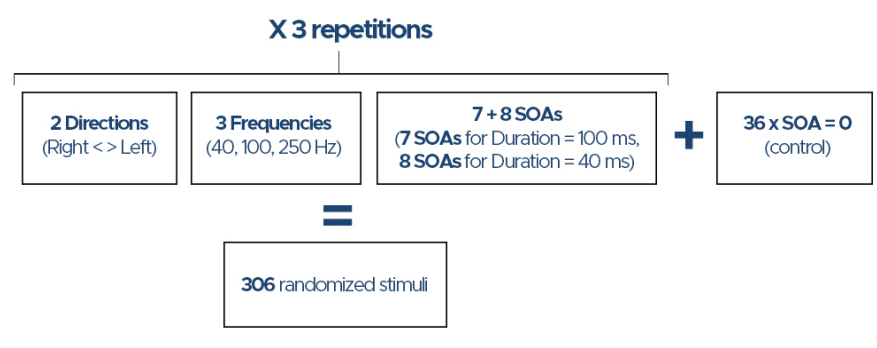

Fig. 6. Experimental design for experiment 2. Every mid-air haptics stimulus was a combination of the four variables (i.e., duration, direction, frequency, SOA), and a control condition with SOA set at 0 , for a total of 306 randomized stimuli. The picture is not representative of the order of presentation of the stimuli.

smooth ATM between the two hands, employing a static point. In the next section, we will discuss the optimal parameters needed when using a dynamic point.

\section{EXPERIMENT 2: TACTILE ILLUSION OF MOVEMENT WITH A DYNAMIC FOCAL POINT}

The aim of this second experiment was to investigate whether using a dynamic point instead of a static focal point on the hands' palm would result in a smoother sensation of movement. For this experiment, we used again the same psychophysical approach used for experiment 1 (see Section IV-A.

\section{A. Method}

This second experiment consisted of 102 randomized tactile stimuli repeated three times, for a total of 306 stimuli. Participants received the same familiarization as in experiment 1 before proceeding to the study phase. An overview of the experimental design and conditions is shown in Fig. 4

The procedure was the same as for experiment 1 , with the key difference that the mid-air tactile stimulus was a dynamic focal tactile point instead of a static one. The focal point was moving along a straight line from one hand to the other (see Fig. 11, being in contact with the participants' palm for a length of $4 \mathrm{~cm}$, with a speed varying according to the duration of the stimulus. During the experiment, every stimulus was a combination of four variables: duration $(100 \mathrm{~ms}$ and 400 $\mathrm{ms}$ ), SOA (two different sets of 8 and 7 intervals, depending on the stimulus's duration, plus the control SOA), frequency $(40 \mathrm{~Hz}, 100 \mathrm{~Hz}$, and $250 \mathrm{~Hz}$ ) and direction (from left to right and vice-versa). Stimuli were presented one at the time, with at least five seconds gap to avoid tactile habituation. Each block was separated by a 2-minute break. Participants wore headphones to mask environmental and device noises, and a beep sound was played through the headphones before the beginning of each trial. Overall, the experiment lasted 50 minutes. All participants were compensated with a $£ 7.5$ voucher for participating in the experiment.

\section{B. Participants}

A total of 20 participants took part in the study ( 9 females, age $\mu=26, \mathrm{SD}= \pm 6.36$ ). They had normal or glasses/lens corrected vision and no history of neurological or psychological disorders. All participants were right-handed. Upon arrival, participants were asked to read the information sheet and sign a consent form, followed by the task explanation.

\section{Results}

To analyze the data, we followed the same procedure as in experiment 1. Fig. 7 illustrates the average ratings as a function of SOA for the two test durations, the two directions, and the three frequencies along with best-fit quadratic trends. The two lowest parts of the curves correspond to low SOAs (merged tactile perception) and to high SOAs (discrete tactile perception). The peaks of the curves correspond to the optimal values of the SOAs and are reported in Table II
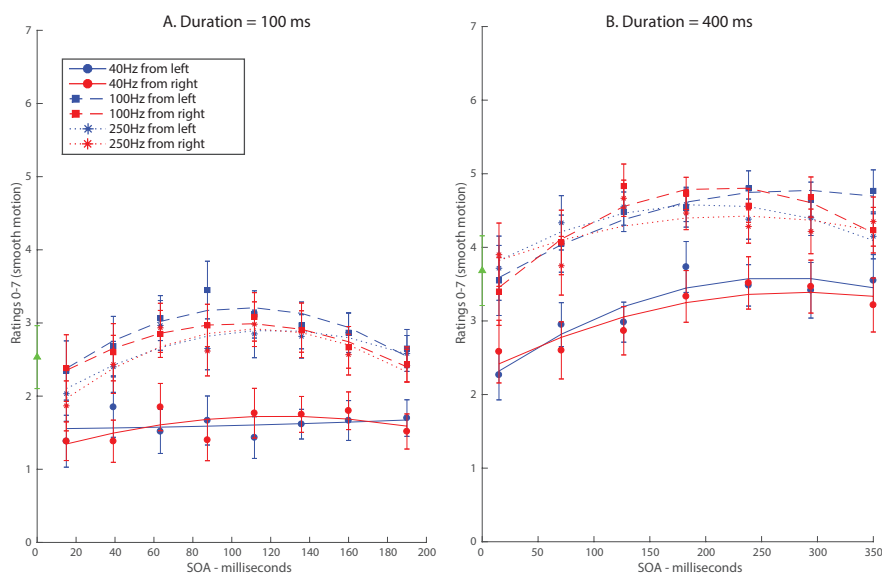

Fig. 7. Plots of the ratings of the illusion of movement (x-axis) per SOAs (y-axis). The left graph shows the plot for $100 \mathrm{~ms}$ duration, and the right graph shows the plot for $400 \mathrm{~ms}$ duration. Dots and lines represent raw data and model fitting, respectively.

On average the optimal SOA value was found to be 175.53. As in experiment 1 , our hypothesis is that with very small and very large values of SOA, participants' rating on the smoothness of the illusion of movement should decrease. Therefore, we fit a quadratic model to describe our dataset: $\mathrm{y}=$ duration $+\mathrm{SOA}+\mathrm{SOA}^{2}$ (model 1). moreover, from Fig 7 , the

TABLE II

OPTIMAL SOA VALUES (IN MS.) AND QUADRATIC FIT $\left(\mathrm{R}^{2}\right)$ FOR THE DIFFERENT COMBINATIONS OF DURATION, FREQUENCY, AND DIRECTION.

\begin{tabular}{lllcl}
\hline Duration & Frequency & Direction & SOA peak (ms.) & $\mathrm{R}^{2}$ \\
\hline $100 \mathrm{~ms}$ & $40 \mathrm{~Hz}$ & from left & 58.49 & .07 \\
$100 \mathrm{~ms}$ & $40 \mathrm{~Hz}$ & from right & 107.34 & .83 \\
$100 \mathrm{~ms}$ & $100 \mathrm{~Hz}$ & from left & 121.75 & .80 \\
$100 \mathrm{~ms}$ & $100 \mathrm{~Hz}$ & from right & 124.48 & .40 \\
$100 \mathrm{~ms}$ & $250 \mathrm{~Hz}$ & from left & 104.20 & .95 \\
$100 \mathrm{~ms}$ & $250 \mathrm{~Hz}$ & from right & 112.95 & .82 \\
$400 \mathrm{~ms}$ & $40 \mathrm{~Hz}$ & from left & 266.78 & .87 \\
$400 \mathrm{~ms}$ & $40 \mathrm{~Hz}$ & from right & 280.84 & .96 \\
$400 \mathrm{~ms}$ & $100 \mathrm{~Hz}$ & from left & 200.79 & .87 \\
$400 \mathrm{~ms}$ & $100 \mathrm{~Hz}$ & from right & 285.47 & .85 \\
$400 \mathrm{~ms}$ & $250 \mathrm{~Hz}$ & from left & 214.88 & .90 \\
$400 \mathrm{~ms}$ & $250 \mathrm{~Hz}$ & from right & 228.40 & .45 \\
\hline
\end{tabular}


curve for the $40 \mathrm{~Hz}$ frequency seems to have obtained a lower rating compared to the other two frequency curves (i.e., the 100 and the $250 \mathrm{~Hz}$ ). Indeed, some participant during the study referred to not being sure of the perception of this frequency because "too weak" and "subtle". Therefore, in our model we treated the variable frequency as categorical and used the 40 $\mathrm{Hz}$ frequency as the baseline. Our model obtained a R2 $=.45$. Following, we report the equations for the $40 \mathrm{~Hz}, 100 \mathrm{~Hz}$, and $250 \mathrm{~Hz}$ frequencies:

2) $y=.66+4 * 10^{-3} * d u r+6 * 10^{-3} * S O A-1.2 * 10^{-5} * S O A^{2}$

3) $y=1.2+4 * 10^{-3} * d u r+6 * 10^{-3} * S O A-1.2 * 10^{-5} * S O A^{2}$

4) $y=1.1+4 * 10^{-3} * d u r+6 * 10^{-3} * S O A-1.2 * 10^{-5} * S O A^{2}$

These results are consistent with the curves' shape showed in Figure 7 (see the intercept term), where the 100 and 250 $\mathrm{Hz}$ frequencies seem to overlap, with the $40 \mathrm{~Hz}$ frequency having the lowest rating scores. In fact, some participants referred uncertainty in perceiving this frequency or described it as really light and hard to perceive.

In summary, based on our two experiments we have created a first-time insight into the use of mid-air haptics for creating a tactile illusion of movement, testing a static versus a dynamic focal point. Following, we first discuss the findings comparing both stimulation approaches, and then we present a discussion comparing our results using mid-air touch against using physical touch in the creation of the ATM. We conclude with a discussion on future works and opportunities for design.

\section{COMPARING STATIC VS. DYNAMIC MID-AIR FOCAL POINTS}

In this section, we are interested in comparing results from experiment 1 (static point) with experiment 2 (dynamic point), to understand how the perception of the ATM is affected by the two different approaches employed in this study.

At a preliminary inspection, the rating curves of experiment 1 and experiment 2 (see Fig. 5 and 7) appear different, with the curves of experiment 1 being sharper, having a clear peak for the optimal SOAs. Moreover, when the SOAs are too short or too long, participants' ratings are clearly decreasing. On the contrary, for experiment 2 , it is harder to visualize the same trend.

We hypothesize that when a dynamic focal point is delivered on the hands (experiment 2), subjects will always perceive a certain amount of movement, that is, the perceptual information will be perceived more confusing compared to a static focal point (experiment 1). In other words, in the dynamic focal point condition, the SOA seems to play a minor role in the delivery of the illusion of movement. This means, that when we want to render a smooth sensation of movement using a dynamic focal point, the SOA is not crucial as for a static focal point.

Next, to compare the results obtained from experiment 1 with those of experiment 2, we estimated the linear and quadratic terms for predicting smoothness ratings from SOA at each duration (100 ms and $400 \mathrm{~ms}$ ) and frequency $(40 \mathrm{~Hz}, 100$ $\mathrm{Hz}$, and $250 \mathrm{~Hz}$ ) for each subject (20). Therefore, we extracted six linear and six quadratic terms for each subject. We checked the distribution of these data through a Shapiro-Wilk test. Then, we run separate independent t-test between the linear and quadratic terms of data from experiment 1 and 2 across duration and frequency. If the distribution of a certain set of data was not following a normal distribution we employed a Mann-Whitney U test for the comparison.

When compared across the two experiments (static vs. moving mid-air point), the linear and the quadratic terms obtained from the quadratic fitting of the ratings of smoothness, led to statistical differences in all cases (all the p-values $<.001$ ). The linear and quadratic coefficient of data from experiment 2 were lower in all cases compared to those of experiment 1 . This demonstrates that the curves from Fig. 7 (experiment 2) are indeed flatter than those from Fig. 5] (experiment 1). This confirms that when we deliver a moving point on the hands, the SOA does not play a fundamental role, and participants tend to rate the smoothness of motion always in the same way. This could confirm that the physical movement provided on the hands and the illusory one are conflated in participants' judgment.

Further, we were interested in understanding if the smoothness of motion's ratings for experiment 2 were higher compared to those of experiment 1 . We calculated the peaks of the curves for the smoothness of motion's ratings for each participant $(\mathrm{N}=20)$, for each duration $(100 \mathrm{~ms}$ and $400 \mathrm{~ms})$, at each frequency $(40 \mathrm{~Hz}, 100 \mathrm{~Hz}$, and $250 \mathrm{~Hz}$ ). Similarly to the previous analyses we used an independent t-test or a MannWhitney $U$ test depending on the shape of data distribution. For the duration $=100 \mathrm{~ms}$ we did not obtain any significant results $(\mathrm{p} .>.05)$. On the contrary, for the duration $=400$ ms, we found two statistical differences: between the $40 \mathrm{~Hz}$ frequencies $(\mathrm{p} .=.01,40 \mathrm{~Hz}$-exp1-mean $=4.8 ; 40 \mathrm{~Hz}$-exp2mean $=3.8)$ and between the $250 \mathrm{~Hz}$ frequencies $(\mathrm{p} .=.05$, $250 \mathrm{~Hz}$-exp1-mean $=4.4 ; 250 \mathrm{~Hz}$-exp2-mean = 5.1).

In lights of these results, we cannot say if experiment 2 achieved a higher illusion of movement. Indeed, as previously stated, the $40 \mathrm{~Hz}$ frequency in experiment 2 was not well perceived, hence, the significant difference. Participants reported the $40 \mathrm{~Hz}$ frequency as too low, too subtle, or too sparse. It might be that the skin sensitivity along the stimulated location is not uniform, and an already subtle frequency will result in a confused percept. For the $250 \mathrm{~Hz}$ instead, the p. value is borderline, and it does not allow for strong conclusions. As this is the first investigation of mid-air tactile stimuli for creating the ATM, further studies are needed to validate our research.

\section{DISCUSSION}

This study investigated, for the first time, the occurrence of the ATM using mid-air haptic technology, comparing a static versus a dynamic tactile focal point. With experiment 1 we established that it is possible to elicit an illusion of movement between two unconnected hands by using a static focal point. We then determined and described the optimal parameters to achieve a smooth tactile illusion of movement using a psychophysical approach. We generated a perceptual 
model that expresses the relation between the duration and the SOA of the tactile stimuli (model 1). This model specifies the optimal parameters to use for achieving a smooth illusion of motion between the hands. The most relevant variables impacting users' perception are the duration and the stimulusonset asynchrony (SOA) of the tactile stimuli, confirming previous results ([3], [4], [36]). In experiment 2, we replicated experiment 1 using a dynamic focal point. We derived a perceptual model (model 2,3,4) for the optimal parameters to achieve a smooth illusion of movement.

To enrich our understanding of creating a tactile illusion of movement, we compared results from experiment 1 and experiment 2 with respect to their effectiveness in achieving a smooth sensation of movement. The results suggest that there is no difference in the perceived smoothness of motion, but using a moving point could inflate the rating of the illusory motion.

\section{LIMITATIONS AND FUTURE WORKS}

Our results indicate that mid-air touch represents a promising approach to deliver an illusion of motion. In this section, we discuss some limitations and challenges of employing midair tactile technology, and we provide ideas for future research.

The mid-air haptic device provides a subtle tactile feedback (like puffs of air, or breeze [35]), and as stated in experiment 2 , low frequencies might constitute a limitation. Previous research showed that the waveform of a tactile stimulus can lower or increase the absolute tactile thresholds (e.g. sinusoidal vs. square) [37]. Future work could investigate the ATM delivering the tactile mid-air stimulus through a different waveform (e.g. square shape) to observe whether the effect of the illusion would be strengthened.

In this study, we investigated the ATM using the device positioned statically on a desk, to set the basis of the understanding of the phenomenon as mediated by mid-air touch. Future work could explore how this illusion of movement would change when the participant is free to move the hands throughout the space.

Our findings and prior work [3], [4] have shown the occurrence of the ATM between the hands. It would be interesting to test, with mid-air but also with physical touch, the possibility of recreating an effect of movement between different parts of the body, e.g. hands and feet, and to observe if the relationship between the durations and the SOA of the tactile stimuli would change. Finally, other technologies could be used to explore the ATM perception, such as wearable devices.

\section{CONCLUSION}

This study investigated, for the first time, the occurrence of the ATM using a mid-air haptic device. We obtained the optimal parameters to achieve a smooth motion using a static versus a dynamic mid-air focal point. We provided a perceptual model for each approach used. We then compared the results obtained from a static versus a dynamic point on the palms. These data suggest no difference between the two approaches, but the first (static point) might be preferable to achieve a clean sensation of motion.
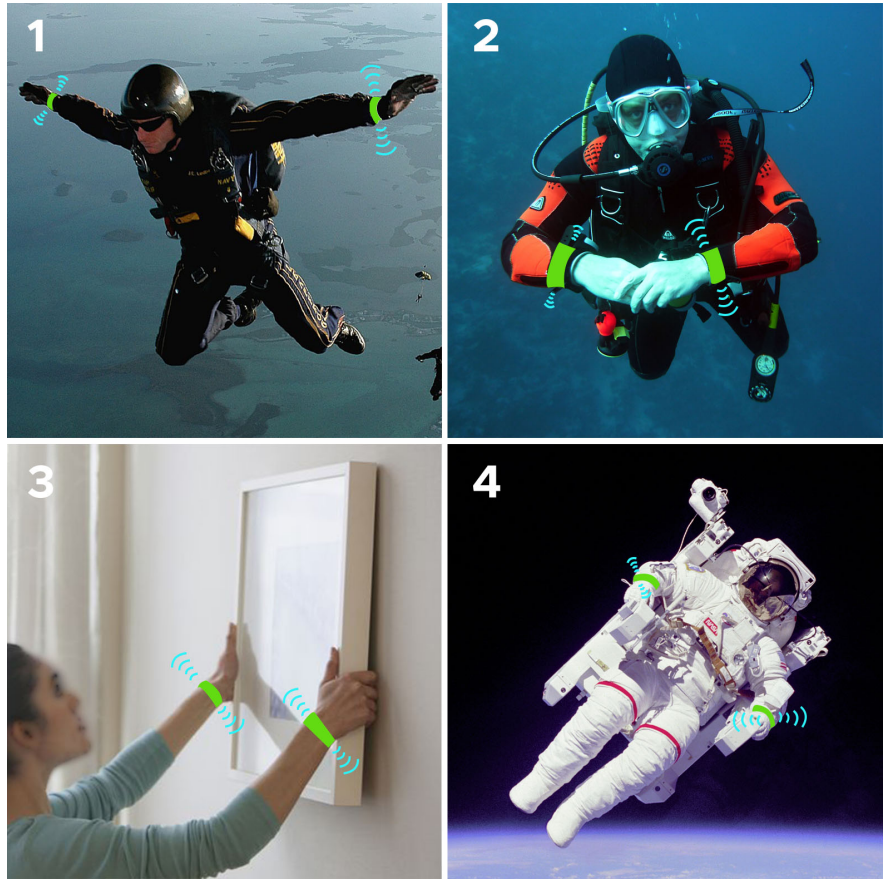

Fig. 8. Example of applications where vision could be unreliable and the tactile apparent motion could provide alternative orientation information. 1) Skydiving: the environment could results visually flat. 2) Underwater: humans can lose orientation underwater. 3) The ATM could be used to provide information on the balance (e.g. when hanging a picture on the wall, when the picture is straight, the motion will not be perceived anymore). 4) In space humans can lose orientation.

Knowing the optimal parameters required to model a smooth sensation of movement can allow for new experiences in VR and non-VR environments. We could now feel the breeze of the wind and the movement of the waves of the sea. In addition, the phenomenon of the ATM could be used to provide directional information (e.g. as a tactile GPS) in cases where visual cues may be unreliable (e.g. in space, underwater, or skydiving) or absent (e.g. in the dark or in the case of the visually impaired population). When underwater, free falling in the sky, or in space, our vision may be unreliable and tactile motion could help to guide us towards our target. Furthermore, this sense of motion could provide hands-free information about the current position and balance of an object we are hanging or carrying (e.g. acting as a tactile bubble level) (Fig. 8).

We believe that this study provides a valuable insight into users' perception of mid-air tactile stimulation, and it will open space for new immersive and realistic scenarios in gaming experiences in VR, AR, and in traditional games.

\section{REFERENCES}

[1] A. Israr and I. Poupyrev, "Tactile Brush : Drawing on Skin with a Tactile Grid Display," Proceedings of CHI'11, pp. 2019-2028, 2011.

[2] A. Israr, S. Zhao, K. Mcintosh, Z. Schwemler, A. Fritz, J. Mars, J. Bedford, C. Frisson, I. Huerta, M. Kosek, B. Koniaris, and K. Mitchell, "Stereohaptics : A Haptic Interaction Toolkit for Tangible Virtual Experiences," ACM SIGGRAPH 2016 Workshop Course 2016, pp. 23, 2016.

[3] S. Zhao, A. Israr, and R. Klatzky, "Intermanual apparent tactile motion on handheld tablets," IEEE World Haptics Conference, WHC 2015, pp. 241-247, 2015. 
[4] D. Pittera, M. Obrist, and A. Israr, "Hand-to-hand: an intermanual illusion of movement," in Proceedings of the 19th ACM International Conference on Multimodal Interaction - ICMI 2017, 2017, pp. 7381. [Online]. Available: http://dl.acm.org/citation.cfm?doid=3136755. 3136777

[5] D. Ablart, C. Velasco, and M. Obrist, "Integrating mid-air haptics into movie experiences," in TVX 2017 - Proceedings of the 2017 ACM International Conference on Interactive Experiences for TV and Online Video, 2017. [Online]. Available: http://delivery.acm.org/10.1145/3080000/3077551/p77-ablart. pdf?ip=139.184.48.84\&id=3077551\&acc $=$ ACTIVESERVICE\&key $=$ BF07A2EE685417C5.93CE57E634C2294D.4D4702B0C3E38B35 4D4702B0C3E38B35\&CFID $=716385572 \&$ CFTOKEN $=23280940 \&$ $\mathrm{acm} \_=1505225856 \_0 \mathrm{c} 7 \mathrm{bad} 3034 \mathrm{ac} 09 \mathrm{f} 3554 \mathrm{dbf}$

[6] C. T. Vi, D. Ablart, E. Gatti, C. Velasco, and M. Obrist, "Not just seeing, but also feeling art: Mid-air haptic experiences integrated in a multisensory art exhibition," International Journal of Human Computer Studies, vol. 108, pp. 1-14, 2017. [Online]. Available: http://ac.els-cdn. com/S1071581917300988/1-s2.0-S1071581917300988-main.pdf? tid=d2eb16f4-97c4-11e7-8046-00000aacb35f\&acdnat $=1505225906$ b35652c3662e8b140524b55c853ddfea

[7] T. Carter, S. A. Seah, B. Long, B. Drinkwater, and S. Subramanian, "UltraHaptics : Multi-Point Mid-Air Haptic Feedback for Touch Surfaces," Proc. UIST 2013, pp. 505-514, 2013.

[8] B. Long, S. A. Seah, T. Carter, and S. Subramanian, "Rendering volumetric haptic shapes in mid-air using ultrasound," $A C M$ Transactions on Graphics, vol. 33, no. 6, pp. 1-10, 2014. [Online]. Available: http://dl.acm.org/citation.cfm?doid=2661229.2661257

[9] M. Obrist, S. Subramanian, E. Gatti, B. Long, and T. Carter, "Emotions Mediated Through Mid-Air Haptics," Proc. CHI'15, pp. 2053-2062, 2015.

[10] Y. Horiuchi, K. Yoshida, Y. Makino, and H. Shinoda, "Rubber hand illusion using invisible tactile stimulus," in 2017 IEEE World Haptics Conference, WHC 2017, no. June, 2017, pp. 490-494.

[11] S. J. Lederman and R. L. Klatzky, "Haptic perception: A tutorial," Attention, perception \& psychophysics, vol. 71, no. 3, pp. 481-489, 2009.

[12] V. Hayward, "A brief taxonomy of tactile illusions and demonstrations that can be done in a hardware store," Brain Research Bulletin, vol. 75, no. 6 , pp. 742-752, 2008

[13] N. Hogan, B. Kay, E. Fasse, and F. Mussa-Ivaldi, "Haptic Illusions: Experiments on Human Manipulation and Perception of \&quot;Virtual Objects\&quot;", Cold Spring Harbor Symposia on Quantitative Biology, vol. 55, no. 0, pp. 925-931, 1 1990. [Online]. Available: http://symposium.cshlp.org/cgi/doi/10.1101/SQB.1990.055.01.086

[14] S. J. Lederman and L. a. Jones, "Tactile and Haptic Illusions," IEEE Transactions on Haptics, vol. 4, no. 4, pp. 273-294, 2011.

[15] M. Hollins and O. Favorov, "The tactile movement aftereffect," Somatosensory \& Motor Research, vol. 11, no. 2, pp. 153-162, jan 1994. [Online]. Available: http://www.tandfonline.com/doi/full/10.3109/ 08990229409028868

[16] H. Lee, S. Member, J.-s. Kim, S. Member, J.-y. Kim, S. Choi, J.-H. Jun, J.-R. Park, A.-h. Kim, S. Member, H.-B. Oh, S. Member, J.-H. Baek, and S.-J. Yang, "Mid-Air Tactile Stimulation Using Indirect Laser Radiation," IEEE Transactions on Haptics, vol. 9, no. 4, pp. 574-585, 10 2016. [Online]. Available: http://ieeexplore.ieee.org/document/7470276/

[17] A. Moscatelli, M. Bianchi, A. Serio, A. Terekhov, V. Hayward, M. O. Ernst, and A. Bicchi, "The Change in Fingertip Contact Area as a Novel Proprioceptive Cue," Current Biology, vol. 26, no. 9, pp. 1159-1163, 2015. [Online]. Available: http://dx.doi.org/10.1016/j.cub.2016.02.052

[18] F. A. Geldard and C. E. Sherrick, "The cutaneous "rabbit": a perceptual illusion." Science (New York, N.Y.), vol. 178 , no. 57, pp. 178-179, 1972. [Online]. Available: http://science.sciencemag.org/content/178/4057/178\%5Cnhttp: //science.sciencemag.org/content/178/4057/178.full.pdf+html\%5Cnhttp: //science.sciencemag.org/content/sci/178/4057/178.full.pdf\%5Cnhttp: //www.ncbi.nlm.nih.gov/pubmed/5076909

[19] G. von Békésy, "Experiments in Hearing," The Journal of the Acoustical Society of America, vol. 88, no. 6, p. 2905, 1960.

[20] J. H. Kirman, "Tactile apparent movement: The effects of interstimulus onset interval and stimulus duration," Perception \& Psychophysics, vol. 15 , no. 1, pp. 1-6, 1974.

[21] H. E. Burtt, "Tactual illusions of movement." Journal of Experimental Psychology, vol. 2, no. 5, pp. 371-385, 1917. [Online]. Available: http://content.apa.org/journals/xge/2/5/371http://psycnet.apa. org/journals/xge/2/5/371/\%5Cnhttp://content.apa.org/journals/xge/2/5/ $371 \mathrm{http}: / /$ psycnet.apa.org/doiLanding?doi=10.1037\%2Fh0074614
[22] E. G. Boring, Sensation and perception in the history of experimental psychology. Appleton Century Crofts, inc., 1942. [Online]. Available: https://books.google.co.uk/books/about/Sensation_and_ Perception_in_the_History.html?id=YldqAAAAMAAJ\&redir_esc=y

[23] C. E. Sherrick and R. Rogers, "Apparent haptic movement," Perception \& Psychophysics, vol. 1, no. 6, pp. 175-180, 1966.

[24] J. H. Kirman, "Tactile apparent movement: The effects of shape and type of motion," Perception \& Psychophysics, vol. 34, no. 1, pp. 96$102,1983$.

[25] M. Miyazaki, M. Hirashima, and D. Nozaki, "The "Cutaneous Rabbit" Hopping out of the Body," Journal of Neuroscience, vol. 30, no. 5, pp. 1856-1860, 2010. [Online]. Available: http://www.jneurosci.org/content/30/5/1856http: //www.jneurosci.org/cgi/doi/10.1523/JNEUROSCI.3887-09.2010

[26] E. C. Lechelt and R. Borchert, "The interdependence of time and space in somesthesis: The Tau effect reexamined," Bulletin of the Psychonomic Society, vol. 10, no. 3, pp. 191-193, 9 1977. [Online]. Available: http://link.springer.com/10.3758/BF03329320

[27] G. Wilson, T. Carter, S. Subramanian, and S. A. Brewster, "Perception of Ultrasonic Haptic Feedback on the Hand: Localisation and Apparent Motion," Proceedings of the 32nd annual ACM conference on Human factors in computing systems - CHI '14, no. October 2015, pp. 1133-1142, 2014. [Online]. Available: http://dl.acm.org/citation.cfm? id=2557033\% 5Cnhttp://dl.acm.org/citation.cfm?doid=2556288.2557033

[28] H. Lee, J. S. Kim, S. Choi, J. H. Jun, J. R. Park, A. H. Kim, H. B. Oh, H. S. Kim, and S. C. Chung, "Mid-air tactile stimulation using laserinduced thermoelastic effects: The first study for indirect radiation," in IEEE World Haptics Conference, WHC 2015, 2015, pp. 374-380.

[29] R. Sodhi, Poupyrev, M. Glisson, and a. Israr, "AIREAL: interactive tactile experiences in free air," $A C M$ Transactions on Graphics, vol. 32, no. 4, p. 134, 2013. [Online]. Available: http://dl.acm.org/citation.cfm?id=2462007\%5Cnpapers: //c80d98e4-9a96-4487-8d06-8e1acc780d86/Paper/p12137

[30] Y. Suzuki and M. Kobayashi, "Air jet driven force feedback in virtual reality," IEEE Computer Graphics and Applications, vol. 25, no. 1, pp. 44-47, 2005.

[31] S. Gupta, D. Morris, S. N. Patel, and D. Tan, "AirWave : Non-Contact Haptic Feedback Using Air Vortex Rings," Ubicomp 2013, pp. 1-11, 2013.

[32] T. Hoshi, T. Iwamoto, and H. Shinoda, "Non-contact tactile sensation synthesized by ultrasound transducers," Proceedings - 3rd Joint EuroHaptics Conference and Symposium on Haptic Interfaces for Virtual Environment and Teleoperator Systems, World Haptics 2009, pp. 256260, 2009.

[33] C. Hatzfeld, Engineering Haptic Devices. Springer, London, 2014. [Online]. Available: http://link.springer.com/10.1007/978-1-4471-6518-7

[34] D. A. Mahns, N. M. Perkins, V. Sahai, L. Robinson, and M. J. Rowe, "Vibrotactile Frequency Discrimination in Human Hairy Skin," Journal of neurophysiology, vol. 95, no. 3, pp. 1442-1450, 2006. [Online]. Available: http://www.ncbi.nlm.nih.gov/pubmed/16319219

[35] M. Obrist, S. A. Seah, and S. Subramanian, "Talking about tactile experiences," in Proceedings of the SIGCHI Conference on Human Factors in Computing Systems - CHI '13. New York, New York, USA: ACM Press, 4 2013, p. 1659. [Online]. Available: http://dl.acm.org/citation.cfm?id=2470654.2466220http://dl.acm.org/ citation.cfm?id=2466220\%5Cnfile:///Users/grahamwilson/Documents/ Papers/2013/Obrist/Obrist2013ProceedingsofCHI2013\%5Cn.pdf\% 5Cnpapers://c80d98e4-9a96-4487-8d06-8e1acc780d86/Paper/p9726

[36] S. Zhao, A. Israr, M. Fenner, and R. Klatzky, "Intermanual Apparent Tactile Motion and its Extension to 3D Interactions," IEEE Transactions on Haptics, vol. 1412, no. c, pp. 1-1, 2017. [Online]. Available: http://ieeexplore.ieee.org/document/7876800/

[37] K. E. MacLean, M. J. Enriquez, and T. Lim, "Morphing in periodic tactile signals," Proceedings - 3rd Joint EuroHaptics Conference and Symposium on Haptic Interfaces for Virtual Environment and Teleoperator Systems, World Haptics 2009, pp. 178-183, 2009. 


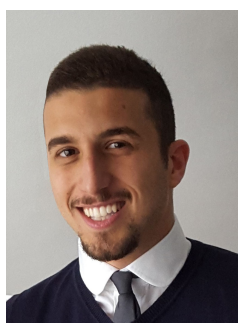

Dario Pittera received his B.S. in Communication and Psychology (2011) and his M.S. in Clinical psychology, developmental psychology and neuropsychology (2014), both from the Universitá di MilanoBicocca, Milan, Italy. He worked as intern at the Ospedale Policlinico di Milano from the 09/2013 to the $08 / 2014$. He was a visiting researcher at the University of Birmingham from 09/2015 to 09/2016, where he was interested in psychophysics and haptic feedback technology. After that he started his $\mathrm{PhD}$ position in the SCHI Lab, School of Engineering and Informatics, at the University of Sussex. From 10/2016 to 02/2017 he worked as Lab Associate at Disney Research, Pittsburgh, PA, US. His research is focused on the psychophysical exploration of mid-air haptic technology and on how to achieve embodiment by using mid-air haptic technology.

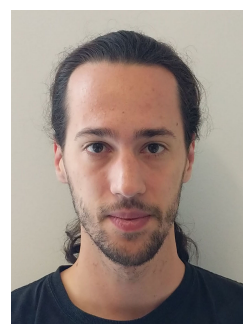

Damien Ablart obtained his M.S. in HumanComputer Interactions and Computer Sciences in September 2015 and his B.S. in Computer Sciences in 2012, both from the University Paul Sabatier (Toulouse III France). During his M.S., he joined the SCHI Lab from March to August 2015 for a research internship. During this time he investigated the use of mid-air haptics for augmenting movie and art experiences. Most notably, he was working on the Tate Sensorium project the creation of a multisensory art display in Tate Britain. In November 2015, he then started his PhD at the SCHI Lab investigating the integration of touch and taste into media, fostering our understanding around emotions and the senses.

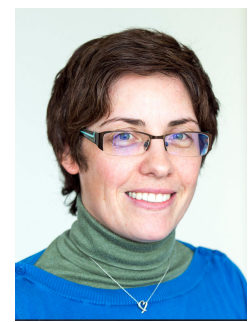

Marianna Obrist is Professor of Multisensory Experiences at the Department of Informatics, School of Engineering and Informatics at the University of Sussex, UK. Marianna is leading the Sussex Computer Human Interaction Lab (SCHI sky Lab), a research group dedicated to the investigation of multisensory experiences for interactive technology. The SCHI Lab team explores tactile, gustatory, and olfactory experiences as novel interaction modalities. Before joining Sussex, Marianna was a Marie Curie Fellow at Newcastle University and prior to this an Assistant Professor for HCI at the University of Salzburg, Austria. More details on her research can be found at: www.multi-sensory.info 